\title{
Simple Evaluation Method for CYP3A4 Induction from Human Hepatocytes: The Relative Factor Approach with an Induction Detection Limit Concentration Based on the $E_{\max }$ Model $^{\circledR}$
}

\author{
Shino Kuramoto, Motohiro Kato, Hidetoshi Shindoh, Akihisa Kaneko, Masaki Ishigai, \\ and Seiji Miyauchi
}

Research Division, Chugai Pharmaceutical Co. Ltd., Kanagawa, Japan (S.K., M.K., H.S., A.K., M.I.); and Department of
Pharmacokinetics, Toho University School of Pharmaceutical Sciences, Chiba, Japan (S.M.)

Received May 15, 2017; accepted August 3, 2017

\section{ABSTRACT}

We investigated the robustness and utility of the relative factor (RF) approach based on the maximum induction effect $\left(E_{\max }\right)$ model, using the mRNA induction data of 10 typical CYP3A4 inducers in cryopreserved human hepatocytes. The RF value is designated as the ratio of the induction detection limit concentration (IDLC) for a standard inducer, such as rifampicin (RIF) or phenobarbital (PB), to that for the compound (e.g., $R F_{\mathrm{RIF}}$ is $I_{\text {IDLC }}$ RIF $/ I D L C_{c p d}$; $R_{\mathrm{PB}}$ is IDLC PB $_{\mathrm{PB}} / \mathrm{IDLC}_{\mathrm{cpd}}$ ). An important feature of the RF approach is that the profiles of the induction response curves on the logarithmic scale remain unchanged irrespective of inducers but are shifted parallel depending on the $\mathrm{EC}_{50}$ values. A key step in the RF approach is to convert the induction response curve by finding the IDLC of a standard inducer. The relative induction score was estimated not only from $E_{\max }$ and $\mathrm{EC}_{50}$ values but also from those calculated by the RF approach. These values showed good correlation, with a correlation coefficient of more than 0.974 , which revealed the RF approach to be a robust analysis irrespective of its simplicity. Furthermore, the relationship between $\mathbf{R F}_{\mathrm{RIF}}$ or $\mathbf{R F}_{\mathrm{PB}}$ multiplied by the steady-state unbound plasma concentration and the in vivo induction ratio plotted using 10 typical inducers gives adequate thresholds for CYP3A4 drugdrug interaction risk assessment. In light of these findings, the simple RF approach using the IDLC value could be a useful method to adequately assess the risk of CYP3A4 induction in humans during drug discovery and development without evaluation of $E_{\max }$ and $\mathrm{EC}_{50}$.

\section{Introduction}

Because a number of drugs have been withdrawn from the market as a result of a serious or fatal drug-drug interaction (DDI) that was found postmarketing, the risk of pharmacokinetic DDIs is considered an important issue that should be given priority in drug development. A DDI is usually caused as a result of inhibition and induction of the family of metabolic enzymes known as cytochrome P450 (P450) enzymes. The induction of $\mathrm{P} 450$ enzymes elicits a remarkable decrease in drug exposure in clinical practice, which results in a reduced therapeutic effect or increased concentrations of active metabolites. Of all P450 enzymes, CYP3A4 is the most abundant in the human liver and small intestine, accounting for $30 \%$ and $80 \%$, respectively, and it plays a major role in the metabolism of approximately $30 \%$ of clinical drugs (Shimada et al., 1994; Thelen and Dressman, 2009; Ohtsuki et al., 2012; Zanger and Schwab, 2013). Thus, evaluating a DDI that arises from CYP3A4 induction in the clinical setting is also a crucial step in drug development.

https://doi.org/10.1124/dmd.117.076349.

S This article has supplemental material available at dmd.aspetjournals.org.
Recently, major pharmaceutical regulatory agencies-the U.S. Food and Drug Administration (FDA), European Medicines Agency (EMA), and the Ministry of Health, Labor and Welfare of Japan-issued guidance or guidelines on investigating DDIs (http://www.ema. europa.eu/docs/en_GB/document_library/Scientific_guideline/2012/ 07/WC500129606.pdf, https://www.fda.gov/downloads/drugs/guidances/ ucm292362.pdf, https://www.solvobiotech.com/documents/Japanese_ DDI_guideline_(draft)_2014Jan.pdf). These documents state the in vitro and in vivo DDI studies that should be conducted during drug development to investigate the inhibition and/or induction of CYP3A4. The studies on DDI mediated by CYP3A4 inhibition are well enough established to give a robust prediction of DDIs in the clinical setting. However, although studies using the human cultured hepatocyte assay system are recommended for CYP3A4 induction, a standard method for quantitatively predicting the DDI mediated by CYP3A4 induction from in vitro data has not yet been accepted.

Thus far, we have demonstrated that using in vitro CYP3A4 induction data from cryopreserved human hepatocytes to quantitatively predict CYP3A4 induction in humans is possible, and we also reported a method of predicting CYP3A4 induction risk in the clinical setting (Kato et al.,

ABBREVIATIONS: AUC, area under the concentration-time curve; $C_{\mathrm{ss}, \mathrm{u}}$, average steady-state unbound plasma concentration; $\mathrm{CL}_{\text {int }}$ hepatic intrinsic clearance; CRB, carbamazepine; DDI, drug-drug interaction; DMSO, dimethylsulfoxide; DXM, dexamethasone; EFV, efavirenz; $E_{\text {max }}$, maximum induction effect; FDA, U.S. Food and Drug Administration; $f_{p}$, unbound fraction in plasma; $f_{u \text {,medium }}$, unbound fraction of a compound in the culture medium; IDL, induction detection limit; IDLC, induction detection limit concentration; LC, liquid chromatography; MS/MS, tandem mass spectrometry; NFD, nifedipine; OMP, omeprazole; P450, cytochrome P450; PB, phenobarbital; PBS, phosphate-buffered saline; PLC, pleconaril; PNT, phenytoin; RF, relative factor; RIF, rifampicin; RIS, relative induction score; SLF, sulfinpyrazone; $S_{\text {LOPE }}$, initial slope of induction response curve of a compound; TRG, troglitazone. 
2005; Kaneko et al., 2010). This prediction method is called the relative factor (RF) approach and uses the maximum induction effect $\left(E_{\max }\right)$ model to evaluate induction of CYP3A4 activity in human hepatocytes. It is important to note that in the $E_{\max }$ model, the profiles of the induction response curves on the logarithmic scale remain unchanged by different inducers but are shifted parallel depending on the $\mathrm{EC}_{50}$ values (Fig. 1A). Therefore, a key step in the RF approach is to convert the induction response curve by the induction detection limit concentration (IDLC) of standard inducers, such as rifampicin (RIF) or phenobarbital (PB). Because of these features, the potential of new chemical entities to induce CYP3A4 can be assessed using only their IDLC values. Other approaches to evaluate CYP3A4 induction from in vitro induction data have been postulated, which use various parameters such as the relative induction score (RIS), $C_{\text {max }} / \mathrm{EC}_{50}$, and area under the concentration-time curve (AUC)/EC $\mathrm{EC}_{50}$ (Persson et al., 2006; Ripp et al., 2006; Chu et al., 2009). These approaches critically require values for the ratio of concentration to CYP3A4 induction response or for induction saturation at higher concentration levels. However, it is difficult to adequately evaluate the induction parameters of new chemical entities that have cytotoxicity, low solubility, or bell-shaped induction (from complex induction and downregulation profiles) (Zhang et al., 2014; Vermet et al., 2016). In contrast, the RF approach circumvents the above problems by evaluating the induction potential of new chemical entities from their minimal concentrations, using the IDLC value (Kaneko et al., 2009).

In this study, we verified the robustness and utility of the RF approach using the induction data of 10 typical CYP3A4 inducers in cryopreserved human hepatocytes taken from three donors. By the RF approach, we evaluated the potential of CYP3A4 induction at the mRNA level as well as in enzymatic activity. The results demonstrate that the simple RF approach using the IDLC value is a useful method that adequately assesses the risk of CYP3A4 induction in humans.

\section{Materials and Methods}

Chemicals. RIF, carbamazepine (CRB), phenytoin (PNT), efavirenz (EFV), pleconaril (PLC), sulfinpyrazone (SLF), nifedipine (NFD), Williams' E medium, bovine serum albumin, gentamicin, ITS+3 liquid media supplement, and HEPES were purchased from Sigma-Aldrich Co. (St. Louis, MO). Dexamethasone (DXM) was purchased from Junsei Chemical Co. Ltd. (Tokyo, Japan). Omeprazole (OMP), PB, dimethylsulfoxide (DMSO), acetonitrile, formic acid, Hanks' balanced salt solution, and phosphate-buffered saline (PBS) were purchased from Wako Pure Chemical Industries Ltd. (Osaka, Japan). Cryopreserved hepatocytes from donors 1 and 2 were purchased from Biopredic International (Rennes, France) (donor 1 was lot no. NON from a 35-year-old Caucasian woman; donor 2 was lot no. IZT from a 44-year-old Caucasian woman) and those from donor 3 were from BioreclamationIVT (New York, NY) (donor 3 was lot no. HEP187075 from a 64-year-old Caucasian man). The selection criterion for donors was that the increase in CYP3A4 at $10 \mu \mathrm{M}$ RIF must be more than 5-fold that without an inducer. The thawing medium and seeding medium were purchased from Biopredic International. Matrigel was purchased from BD Biosciences (Franklin Lakes, NJ). All other chemicals used were of the highest grade available from Wako Pure Chemical Industries Ltd.

Cell Culture. Cryopreserved human hepatocytes were thawed immediately in the thawing medium and centrifuged at $200 \mathrm{~g}$ for 1 minute. The cells were resuspended in the seeding medium and were seeded at $4.0 \times 10^{4}$ cells/well in 96-well plates precoated with collagen type I. After 24-hour incubation, the hepatocyte seeding medium was exchanged with a culture medium composed of Williams' E medium supplemented with $84 \mu \mathrm{g} / \mathrm{ml}$ gentamicin, $1 \% \mathrm{ITS}+3$ liquid media supplement, $1.25 \mathrm{mg} / \mathrm{ml}$ bovine serum albumin, $2 \mathrm{mM}$ glutamine, $10 \mathrm{mM}$ PBS, and $50 \mu \mathrm{g} / \mathrm{ml}$ Matrigel. The cells were maintained in a humidified incubator at $37^{\circ} \mathrm{C}\left(5 \% \mathrm{CO}_{2}\right)$ for 48 hours before $\mathrm{CYP} 3 \mathrm{~A} 4$ induction experiments began.

CYP3A4 Induction Experiment. The concentration ranges for the inducers in the CYP3A4 induction experiments were as follows: RIF, 0.01-10 $\mu \mathrm{M}$; CRB, 3-300 $\mu \mathrm{M}$; PNT, 1-100 $\mu \mathrm{M}$; EFV, 0.1-10 $\mu \mathrm{M}$; PLC, 0.1-100 $\mu \mathrm{M}$; SLF, 1-100 $\mu \mathrm{M}$; DXM, 3-300 $\mu \mathrm{M}$; NFD, 0.1-30 $\mu \mathrm{M}$; PB, 30-1000 $\mu \mathrm{M}$; and OMP, $0.3-30 \mu \mathrm{M}$. All inducers dissolved in DMSO were diluted 1000 -fold with incubation medium [final DMSO concentration, $0.1 \%(\mathrm{v} / \mathrm{v})$ ] before being used in the CYP3A4 induction experiment. The cells were incubated in the culture medium containing an inducer or a vehicle control [0.1\% (v/v) DMSO]. The medium was exchanged with fresh medium containing the inducer or vehicle control every 24 hours. After induction for 72 hours, the cells were washed twice with ice-cold Hanks' balanced salt solution. The cells were lysed using a QuantiGene sample processing kit for cultured cells from Affymetrix (Santa Clara, CA) and were stored at $-80^{\circ} \mathrm{C}$ until mRNA levels were determined.

Determination of the mRNA Level. The mRNA level in the cells was determined according to the instructions provided by Affymetrix. The cell lysates $(50 \mu \mathrm{l})$ were diluted with $30 \mu \mathrm{l}$ lysis mixture and were mixed with a $20-\mu \mathrm{l}$ working plex kit (panel 12117, CYP3A4, and glyceraldehyde 3-phosphate dehydrogenase as a housekeeping gene). Detection of the mRNA level was performed on a Bio-Plex 200 from Bio-Rad (Berkeley, CA).

Determination of Inducer Concentration in the Culture Medium. Each test compound was analyzed by liquid chromatography (LC) coupled with tandem mass spectrometry (MS/MS) as follows. Three-microliter aliquots of the culture medium were added to $297-\mu 1$ internal standard solutions containing $200 \mathrm{nM}$ warfarin and verapamil in acetonitrile. The mixtures were centrifuged for 3 minutes at $10,000 \mathrm{~g}$, and supernatants were injected into the LC-MS/MS system. The LC-MS/MS equipment consisted of a high-performance LC system from Shimadzu (Tokyo, Japan), two LC-30AD pumps, a Nexera autosampler (Nexera Inc., New York, NY), and a CTO-20A column oven coupled with a QTRAP 5500 MS system from AB Sciex (Framingham, MA). A Capcell Core ADME column $(2.7 \mu \mathrm{m}, 50 \times 2.1 \mathrm{~mm}$; Shiseido, Tokyo, Japan $)$ was used as the analytical column. The mobile phase for the analytical column consisted of two solvents: $0.1 \%$ formic acid in water and $0.1 \%$ formic acid in acetonitrile. The flow rate was set at $0.6 \mathrm{ml} / \mathrm{min}$.

Determination of the Unbound Fraction of Each Inducer in the Culture Medium. The unbound fraction of each inducer in the culture medium was determined using a Single-Use RED Plate for rapid equilibrium dialysis (Pierce Biotechnology, Rockford, IL). Three-hundred microliters of the culture medium and $500 \mu 1$ PBS buffer were put into each chamber, respectively. The device was vigorously shaken and incubated at $37^{\circ} \mathrm{C}$. After equilibrium was achieved at $37^{\circ} \mathrm{C}$
A

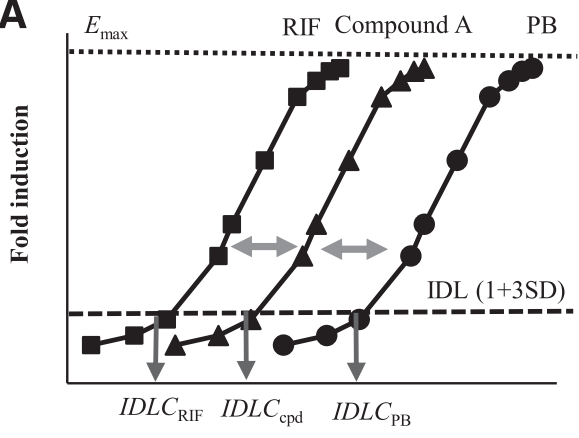

Inducer concentration (logarithmic)

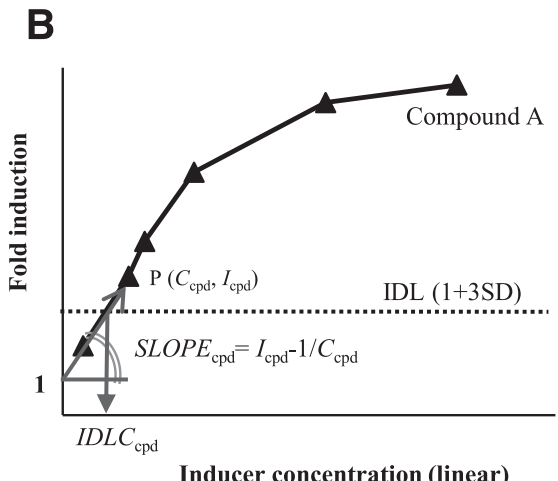

Inducer concentration (linear)
Fig. 1. Conceptual diagram of the RF approach. (A) Curves representing the logarithmic expression of the in vitro induction response to inducer concentration. RIF and PB were used as standard inducers. (B) Curves representing the linear expression of the in vitro induction response to inducer concentration. $I_{\text {cpd }}$, induction response at point $\mathrm{P} ; \mathrm{P}$, the lowest concentration to show an induction response that exceeded the IDL. 
(in a 4-hour dialysis), the concentration of the inducer in both chambers was measured by LC-MS/MS analysis, as described above. The unbound fractions of inducers in the cell culture medium $\left(f_{\mathrm{u}}\right.$, medium $)$ are summarized in Supplemental Table 3.

Estimation of the Average Concentration of Inducers in the Culture Medium. Twenty-microliter aliquots were taken from the culture medium at designated times ( 24 and 48 hours) after the induction experiment began. The concentration of inducers in the culture medium was measured using LC-MS/MS. The time curve for the area under the concentration in medium $\left(\mathrm{AUC}_{24-48}\right)$ was estimated according to the log-linear trapezoidal rule. The average concentration during the 24-hour period $\left(C_{\text {ave }}\right)$ was estimated by dividing $\mathrm{AUC}_{24-48}$ by 24 hours.

Evaluation of RF of the Induction from the IDLC Values. The RF values were calculated as previously described (Kaneko et al., 2009). The level at which the enzyme is significantly induced, called the induction detection limit (IDL), is at 3-fold S.D. from the control level. Therefore, the IDLC value represents the lowest concentration of the compound that significantly induces the enzyme. The initial slope of the induction response curve of a compound ( $\left.\mathrm{SLOPE}_{\mathrm{cpd}}\right)$ was calculated using the lowest concentration $\left(C_{\mathrm{cpd}}\right)$ to show an induction response that exceeded the IDL as follows:

$$
\mathrm{SLOPE}_{\mathrm{cpd}}=\frac{I_{\mathrm{cpd}}-1}{C_{\mathrm{cpd}}}
$$

where $I_{\mathrm{cpd}}$ represents the fold induction of a compound at $C_{\mathrm{cpd}}$ (Fig. 1B).

The induction curve of a compound is a line for which intercept and slope are 1 and $\mathrm{SLOPE}_{\mathrm{cpd}}$, respectively. Therefore, the IDLC value of a compound $\left(\mathrm{IDLC}_{\mathrm{cpd}}\right)$ is calculated by the following equation:

$$
\mathrm{IDLC}_{\mathrm{cpd}}=\frac{\mathrm{IDL}-1}{\mathrm{SLOPE}_{\mathrm{cpd}}}=\frac{3 \mathrm{~S} . \mathrm{D} .}{\mathrm{SLOPE}_{\mathrm{cpd}}}
$$

The RF values for CYP3A4 induction were estimated from the IDLC values of standard inducers $\left(\mathrm{IDLC}_{\mathrm{i}}\right.$ ) as follows:

$$
\mathrm{RF}_{\mathrm{i}}=\frac{\mathrm{IDLC}_{\mathrm{i}}}{\mathrm{IDLC}_{\mathrm{cpd}}}
$$

$\mathrm{RF}_{\mathrm{i}}$ represents the $\mathrm{RF}$ values estimated with the standard inducer i.

In the RF approach, assuming that the $E_{\max }$ values of CYP3A4 induction remain unchanged irrespective of inducers and that induction response curves are shifted parallel depending on the $\mathrm{EC}_{50}$ values (Fig. $1 \mathrm{~A}$ ), $\mathrm{RF}_{\mathrm{i}}$ is calculated by the following equation using $\mathrm{EC}_{50}$ values:

$$
\mathrm{RF}_{\mathrm{i}}=\frac{\mathrm{IDLC}_{\mathrm{i}}}{\mathrm{IDLC}_{\mathrm{cpd}}}=\frac{\mathrm{EC}_{50 \mathrm{i}}}{\mathrm{EC}_{50 \mathrm{cpd}}}
$$

where $\mathrm{EC}_{50 \mathrm{i}}$ is the $\mathrm{EC}_{50}$ of a standard inducer and $\mathrm{EC}_{50 \text { cpd }}$ is the $\mathrm{EC}_{50}$ of a compound.

Prediction of Induction Risk of CYP3A4 in Clinical Settings Using the RF Approach. The average steady-state unbound plasma concentration of a compound $\left(C_{\mathrm{ss}, \mathrm{u}}\right)$ in clinical settings was calculated for each compound, as previously reported (Kato et al., 2005; Ripp et al., 2006). $C_{\mathrm{ss}, \mathrm{u}}$ is estimated by the following equation:

$$
C \text { ss, } \mathrm{u}=\frac{f_{\mathrm{p}} \times F \times \text { Dose }}{\mathrm{CL}_{\mathrm{tot}} \times \tau}=\frac{f_{\mathrm{p}} \times \mathrm{AUC}_{\mathrm{p}}}{\tau}
$$

where $f_{\mathrm{p}}$ is the unbound fraction in plasma, $\mathrm{AUC}_{\mathrm{p}}$ is the area under the plasma concentration-time curve, $\tau$ is the dosage interval, $\mathrm{CL}_{\text {tot }}$ is the total clearance, and $F$ is the bioavailability. The kinetic parameters in eqs 5 and 6 were derived from the database in Goodman and Gilman's The Pharmacological Basis of Therapeutics. $\mathrm{CL}_{\mathrm{tot}}$ was calculated by the following equation:

$$
\mathrm{CL}_{\text {tot }}=\frac{\text { Dose }}{\mathrm{AUC}_{\mathrm{p}}}=\frac{0.693 \times V_{\mathrm{d}}}{T_{1 / 2}}
$$

The hepatic clearance $\left(\mathrm{CL}_{\mathrm{h}}\right)$ was estimated from $\mathrm{CL}_{\mathrm{tot}}$ and the renal clearance $\left(\mathrm{CL}_{\mathrm{r}}\right)$ using the following equation:

$$
\mathrm{CL}_{\mathrm{h}}=\mathrm{CL}_{\mathrm{tot}}-\mathrm{CL}_{\mathrm{r}}
$$

The hepatic intrinsic clearance $\left(\mathrm{CL}_{\text {int }}\right)$ was estimated by eq. 8 based on the well stirred model:

$$
\mathrm{CL}_{\text {int }}=\frac{R_{\mathrm{b}} \times Q_{\mathrm{h}} \times \mathrm{CL}_{\mathrm{h}}}{f_{\mathrm{p}} \times\left(Q_{\mathrm{h}}-\mathrm{CL}_{\mathrm{h}}\right)}
$$

$R_{\mathrm{b}}$ (blood-to-plasma concentration ratio) and $Q_{\mathrm{h}}$ (blood flow rate) were assumed to be 1 and $1610 \mathrm{ml} / \mathrm{min}$, respectively (Kato et al., 2005).

The in vivo induction ratio of CYP3A4 is estimated as the ratio of $\mathrm{CL}_{\text {int }}$ with or without treatment of an inducer under in vivo conditions (Supplemental Table 1). The concentration response of each inducer is converted by multiplying $C_{\mathrm{ss}, \mathrm{u}}$ by $\mathrm{RF}_{\mathrm{i}}$ values. We designated the threshold of the in vivo induction response of CYP3A4 as 1.25 , which corresponds to a $20 \%$ decrement in the AUC of substrates, as designated by the FDA, EMA, and Ministry of Health, Labor and Welfare of Japan. To categorize inducers as having a negative or positive possibility of inducing CYP3A4, we first plotted the induction response curve of CYP3A4 against the converted $C_{\mathrm{ss}, \mathrm{u}}\left(C_{\mathrm{ss}, \mathrm{u}} \times \mathrm{RF}_{\mathrm{i}}\right)$ for each inducer and we found the first and last data points above and below the 1.25 level, which we called "point above 1.25" and "point below 1.25," respectively. Then, a lower threshold was assigned at the data point immediately preceding the point above 1.25 , and an upper threshold was assigned at the data point immediately after the point below 1.25 (Fig. 4). Induction response values that fell below the lower threshold were categorized as negative for a risk of CYP3A4 induction, whereas values that fell above the upper threshold were categorized as positive. Thus, the intervening region, which included compounds with induction responses around the 1.25 level, included false-positive and false-negative compounds.

Calculation of RIS Using the $\boldsymbol{E}_{\max }$ Model and RF Approach. Induction responses of CYP3A4 followed the $E_{\max }$ model. There are several methods of assessing the risk of CYP3A4 induction in clinical settings. RIS correlation, which is recommended in the EMA guideline for risk assessment, is an approach that evaluates the risk of CYP3A4 induction in vivo from in vitro induction data. RIS is calculated by the following equation:

$$
\mathrm{RIS}=\frac{E_{\max } \times[I]}{\mathrm{EC}_{50}+[I]}
$$

where $[I]$ is the unbound maximum plasma concentration.

In RF analysis using RIF as a standard inducer, the $E_{\max }$ of a compound is equal to the $E_{\max }$ of RIF, and the $\mathrm{EC}_{50}$ of a compound is calculated from the $\mathrm{EC}_{50}$ of RIF divided by $\mathrm{RF}_{\mathrm{RIF}}$. $\mathrm{RIS}_{\mathrm{RF}}$ was defined as the RIS that was calculated using these parameters by the following equation:

$$
\mathrm{RIS}_{\mathrm{RF}}=\frac{E_{\operatorname{maxRIF}} \times[I]}{\mathrm{EC}_{50 \mathrm{RIF}} / \mathrm{RF}_{\mathrm{RIF}}+[I]}
$$

To evaluate the adaptability of the RF approach to the $E_{\max }$ model, the values for RIS $_{\mathrm{RF}}$ were compared with those for RIS that were calculated using $E_{\max }$ and $\mathrm{EC}_{50}\left(\mathrm{RIS}_{E \max }\right)$

\section{Results}

Time Profiles of the Concentrations of Test Inducers and Their Stability in the Cell Cultures. The stability of test inducers was determined by measuring their concentration in the culture medium at 24 and 48 hours. RIF, CRB, SLF, DXM, and PB showed good stability with more than $80 \%$ (the percentage of the initial amount) remaining. On the other hand, the remaining amount of NFD was minimal $(1.8 \%$ already at 24 hours) (Supplemental Table 2A). The rest of the test inducers (PNT, EFV, PLC, and OMP) showed moderate stability, which ranged from $25 \%$ to $80 \%$. The $f_{\text {u,medium }}$ values of test inducers were also determined to be between 0.145 and 1.02 (Supplemental Table 3), and the estimated average unbound concentration of inducers is summarized in Supplemental Table 2B.

Evaluation of the CYP3A4 Induction Assay on the mRNA and Enzyme Activity Scales Using Cryopreserved Human Hepatocytes. As shown in Fig. 2, the induction response of CYP3A4 at the mRNA level correlated well with enzyme activity $(r \geq 0.866)$; most of the data 

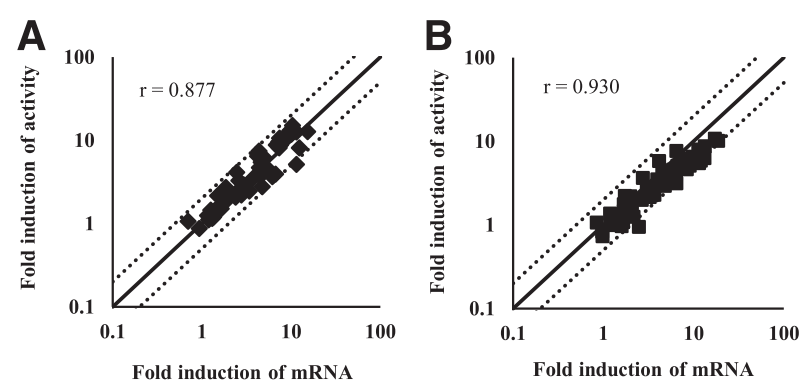

Fig. 2. (A-C) Correlation between CYP3A4 induction at the mRNA level and enzymatic activity in three lots of human hepatocytes [donor 1 (A), donor 2 (B), and donor $3(\mathrm{C})$ ]. Solid lines represent unity and dotted lines represent 2-fold the line of unity. (more than 92\%) fell within the 2-fold line of unity, irrespective of the hepatocyte lot. The relationship between concentration and CYP3A4 induction at the mRNA level is shown in Fig. 3. CYP3A4 was induced in all three lots of human hepatocytes in line with the increase in test inducer concentration, whereas the maximum response of CYP3A4 induction showed variability among donors, with the response in the lot from donor 3 being lower than that in other lots. The induction parameters were estimated using the $E_{\max }$ model and are summarized in Table 1 , which shows that the $E_{\max }$ values deviated widely. The values for parameters estimated in this study (Table 1) ranged within the values reported by others, indicating that the estimates were comparable with others. To standardize the induction response in the three lots of hepatocytes, the IDLC values for all inducers were determined. The thresholds for detection of the induction response were a little different between hepatocyte lots (donor 1, 1.22; donor 2, 1.71; and donor 3, 1.81), because the IDL was designated as 3-fold the S.D. of the experimental data. Utilizing RIF and $\mathrm{PB}$ as standard inducers, $\mathrm{RF}_{\mathrm{RIF}}$ and $\mathrm{RF}_{\mathrm{PB}}$ values were estimated and are summarized in Table 1.

Prediction of CYP3A4 Induction Risk in Humans: The Relationship between $\boldsymbol{C}_{\mathrm{ss}, \mathrm{u}} \times \mathbf{R F}$ and the In Vivo Induction Ratio in Humans. $C_{\mathrm{ss}, \mathrm{u}}$ values were estimated from clinical data by eq. 5 and
$\mathrm{CL}_{\text {int }}$ values were estimated by eq. 8 , using AUC in the clinical setting. We then evaluated the in vivo conversion concentrations of inducers under steady-state conditions $\left(C_{\mathrm{ss}, \mathrm{u}} \times \mathrm{RF}_{\mathrm{i}}\right)$ using the $\mathrm{RF}_{\mathrm{RIF}}$ or $\mathrm{RF}_{\mathrm{PB}}$ value. The ratio of $C_{\mathrm{ss}, \mathrm{u}} \times \mathrm{RF}_{\mathrm{i}}$ of typical CYP3A4 inducers to induction in $\mathrm{CL}_{\text {int }}$ under in vivo conditions is shown in Fig. 4 and Supplemental Table 1 . The features of the induction response calculated via $C_{\mathrm{ss}, \mathrm{u}} \times \mathrm{RF}_{\mathrm{i}}$ values in all lots of human hepatocytes showed characteristics of the $E_{\mathrm{max}}$ model isotherm. The $C_{\mathrm{ss}, \mathrm{u}} \times \mathrm{RF}_{\mathrm{i}}$ values for the threshold at which a high potential of CYP3A4 induction was elicited were nearly similar in each lot of human hepatocytes, except for the threshold for RIF in that of donor 2 , for which the $C_{\mathrm{ss}, \mathrm{u}} \times \mathrm{RF}_{\mathrm{i}}$ value was one-third smaller than others $(2 \mathrm{nM})$ (Fig. 4C). Likewise, the threshold of $C_{\mathrm{ss}, \mathrm{u}} \times \mathrm{RF}_{\mathrm{i}}$ representing significant CYP3A4 induction occurred around $1 \mathrm{nM}$.

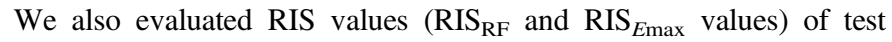
inducers based on the $\mathrm{RF}_{\mathrm{RIF}}$ value and the $E_{\max }$ model, respectively, as explained above by eqs. 9 and 10. The RIS $\mathrm{RF}_{\mathrm{RF}}$ values coincided well with the RIS $_{E \max }$ values in the three lots of human hepatocytes, with correlation coefficients of $0.974,0.986$, and 0.998 in donors 1,2 , and 3 , respectively, and more than $82 \%$ of the relationships ranged within the 3-fold line of unity (Fig. 5). These good correlations indicated the robustness and validity of estimating RIS based on the $\mathrm{RF}_{\mathrm{RIF}}$ values.

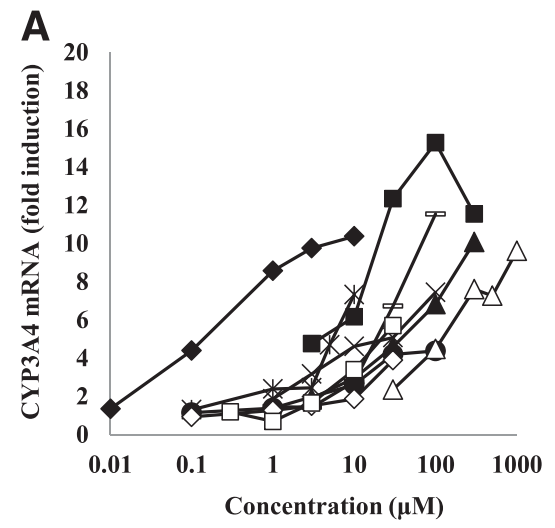

C

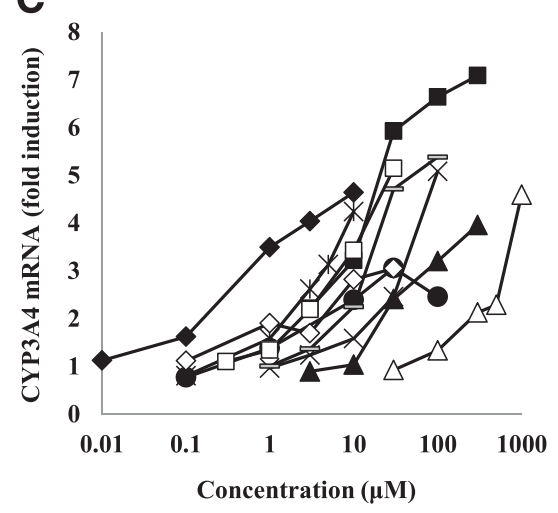

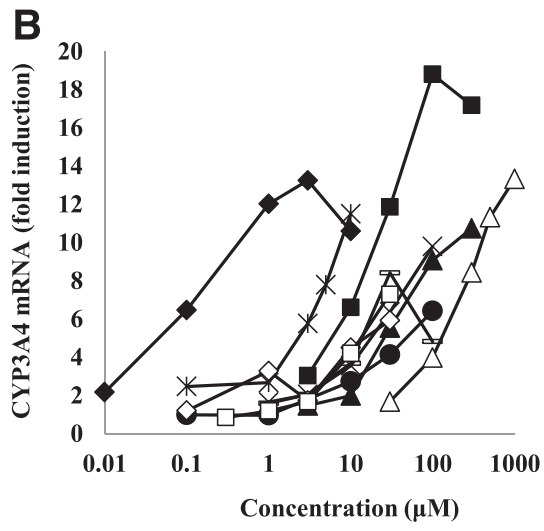

Fig. 3. (A-C) The relationship between inducer concentration and CYP3A4 induction response at the mRNA level for three lots of human hepatocytes [donor 1 (A), donor 2 (B), and donor 3 (C)]. Closed diamond, RIF; closed triangle, CRB; cross, PNT; asterisk, EFV; closed circle, PLC; horizontal bar, SLF; closed square, DXM; open diamond, NFD; open triangle, PB; and open square, OMP. 
TABLE 1

Induction parameters, $\mathrm{RF}_{\mathrm{RIF}}$, and $\mathrm{RF}_{\mathrm{PB}}$ of 10 inducers for three lots of human hepatocytes

$E_{\max }$ and $\mathrm{EC}_{50}$ were estimated by a simple $E_{\max }$ model with WinNonlin 7.0 (Certara, Princeton, NJ).

\begin{tabular}{|c|c|c|c|c|c|c|c|c|c|c|c|c|}
\hline \multirow{2}{*}{ Inducer } & \multicolumn{4}{|c|}{ Donor 1} & \multicolumn{4}{|c|}{ Donor 2} & \multicolumn{4}{|c|}{ Donor 3} \\
\hline & $E_{\max }$ & $\mathrm{EC}_{50}$ & $\mathrm{RF}_{\mathrm{RIF}}$ & $\mathrm{RF}_{\mathrm{PB}}$ & $E_{\max }$ & $\mathrm{EC}_{50}$ & $\mathrm{RF}_{\mathrm{RIF}}$ & $\mathrm{RF}_{\mathrm{PB}}$ & $E_{\max }$ & $\mathrm{EC}_{50}$ & $\mathrm{RF}_{\mathrm{RIF}}$ & $\mathrm{RF}_{\mathrm{PB}}$ \\
\hline RIF & 9.36 & 0.189 & 1 & 790 & 11.4 & 0.0961 & 1 & 5100 & 3.71 & 0.543 & 1 & 1160 \\
\hline CRB & 10.7 & 80.7 & 0.00836 & 6.60 & 11.9 & 47.5 & 0.00099 & 5.04 & 3.75 & 52.4 & 0.0089 & 10.4 \\
\hline PNT & 6.18 & 9.3 & 0.0246 & 19.4 & 12.0 & 39.4 & 0.00491 & 25.0 & 15.9 & 291 & 0.0125 & 14.5 \\
\hline EFV & 36.7 & 50.0 & 0.0841 & 66.4 & 25.5 & 15.5 & 0.126 & 640 & 5.88 & 7.57 & 0.132 & 153 \\
\hline PLC & 3.91 & 11.9 & 0.0175 & 13.8 & 7.45 & 36.7 & 0.00127 & 6.47 & 1.98 & 2.43 & 0.0397 & 46.1 \\
\hline SLF & 18.0 & 68.0 & 0.0131 & 10.4 & 6.14 & 6.62 & 0.00404 & 20.6 & 5.84 & 21.5 & 0.0291 & 33.7 \\
\hline DXM & 13.2 & 9.21 & 0.0350 & 27.6 & 19.2 & 19.9 & 0.00579 & 29.5 & 6.54 & 14.0 & 0.0950 & 110 \\
\hline NFD & 16.9 & 150 & 0.00696 & 5.49 & 6.47 & 12.7 & 0.0190 & 96.8 & 2.26 & 4.32 & 0.213 & 247 \\
\hline PB & 9.71 & 179 & 0.00127 & 1 & 18.1 & 396 & 0.00020 & 1 & 21.1 & 5000 & 0.00086 & 1 \\
\hline OMP & 9.96 & 31.0 & 0.00295 & 2.33 & 13.3 & 30.9 & 0.00197 & 10.0 & 6.06 & 14.1 & 0.0904 & 105 \\
\hline
\end{tabular}

However, RIS $\mathrm{RF}_{\mathrm{RF}}$ values were estimated on the assumption that the $E_{\max }$ value remained constant irrespective of the type of inducer. In the case of a partial agonist, the $E_{\max }$ value would be lower than that of a full agonist and would result in an overestimation of the $E_{\max }$ value and an underestimation of the $\mathrm{EC}_{50}$ value by the $\mathrm{RF}$ approach. The estimated RIS $_{\mathrm{RF}}$ values showed a tendency to be overestimated, which should make us more cautious about interpreting the estimated RIS $_{\mathrm{RF}}$ values.

\section{Discussion}

This study validates the RF approach, which uses the IDLC value of the inducer to calculate a factor based on the $E_{\max }$ model that can predict the induction potential of a metabolic enzyme. In 10 compounds chosen as typical inducers, we determined the enzymatic activity and the potential CYP3A4 induction at the mRNA level and enzymatic activity and found a good correlation between them (Fig. $2)$. In contrast, the concentration-dependent responses for the induction of CYP3A4 mRNA deviated somewhat between different human hepatocyte lots (Fig. 3; Table 1); namely, the absolute values of $E_{\max }$ and $\mathrm{EC}_{50}$ differed by up to 7-fold and 35-fold, respectively. This deviation may be caused by variability in the potency of an induction response in individual donors or differences in the process of preparing human hepatocytes. We thus advocate normalizing by the RF values to those of the standard inducers RIF and PB to minimize the variability in the induction response. With eq. 3 , the RF values can be estimated from the IDLC values, which are the minimal concentrations at which a significant induction of CYP3A4 can be detected. Because they are based on the $E_{\max }$ model, the RF values can also be defined as the ratio of the $\mathrm{EC}_{50}$ value of a compound to that of the standard inducer (eq. 4).

In drug development, assessing whether a new chemical entity has the potential to induce P450 enzymes is very important. FDA guidance recommends that each company should either set its own threshold for P450 enzyme induction according to the method used or should evaluate the $R_{3}$ value to calculate the extent of decrease in AUC when a metabolic enzyme is induced. The $\mathrm{R}_{3}$ value is calculated using the following equation: $\mathrm{R}_{3}=1 /\left(1+d \times E_{\max } \times[I] /\left(\mathrm{EC}_{50}+[I]\right)\right.$, where $[I]$ is the maximal total systemic inducer concentration in plasma and $d$ is the scaling factor assumed as 1 for the basic model. Determining the $R_{3}$ value requires the entire profile of the concentration-induction curve, which hampers the assessment of induction by compounds with poor solubility and/or toxicity. By contrast, in the case of the RF approach with cryopreserved hepatocytes, we could evaluate the threshold of induction using 10 inducers in the clinical setting, even without a complete concentration-induction curve. Unfortunately, none of the
10 inducers used in this study showed toxicity or poor solubility. To assess whether the method is an improvement over current methods in predicting induction potential requires further investigation that includes various compounds with poor solubility and/or toxicity.

The RF values were used to evaluate the conversion concentration against the standard inducers, and the relationship between the conversion concentration and in vivo induction ratio revealed typical concentration-response curves (Fig. 4). When the CYP3A4 induction risk was assessed using RF values, the upper thresholds of different lots of hepatocytes could be evaluated robustly and relevantly. The unbound concentration at steady state corresponding to $\mathrm{RIF}\left(\mathrm{RF}_{\mathrm{RIF}} \times C_{\mathrm{ss}, \mathrm{u}}\right)$ was $7.58,1.99$, and $7.58 \mathrm{nM}$ for donors 1,2 , and 3 , respectively. The unbound concentration corresponding to $\mathrm{PB}\left(\mathrm{RF}_{\mathrm{PB}} \times C_{\mathrm{ss}, \mathrm{u}}\right)$ was 5.98 , 10.1 , and $8.78 \mu \mathrm{M}$ for donors 1,2 , and 3 , respectively. These thresholds obtained from human cryopreserved hepatocytes are comparable with those we predicted from HepaRG cells in a previous report, implying that the RF approach is a very robust and valid approach for predicting the CYP3A4 induction risk of a new chemical entity and will be useful in reducing differences across experiments and across laboratories (Kaneko et al., 2009). As shown in Fig. 4, the $\mathrm{RF}_{\mathrm{i}} \times C_{\mathrm{ss}, \mathrm{u}}$ values for $30 \mathrm{mg} / \mathrm{kg}$ PB decreased on or after the upper threshold, and the $\mathrm{RF}_{\mathrm{i}} \times$ $C_{\mathrm{ss}, \mathrm{u}}$ values for $40 \mathrm{mg} / \mathrm{kg}$ NFD decreased on or before the lower threshold. These $\mathrm{RF}_{\mathrm{i}} \times C_{\mathrm{ss}, \mathrm{u}}$ values for $30 \mathrm{mg} / \mathrm{kg}$ PB and $40 \mathrm{mg} / \mathrm{kg}$ NFD could be used to easily set the negative and positive thresholds for each individual donor. The simplicity and convenience in the estimation of the induction thresholds might be a cost-benefit of using the RF approach for DDI studies during drug development.

Previously, Kanebratt and Andersson (2008) developed the AUC/ $F_{2}$ approach to assess induction risk using HepaRG cells. AUC/ $F_{2}$ values are estimated by dividing AUC by $F_{2}$, where AUC is obtained from the clinical data of the test inducer and $F_{2}$ is the concentration at which an inducer elicits CYP3A4 mRNA that is 2-fold of the baseline. Although a good correlation between in vitro and in vivo results was obtained using $\mathrm{AUC} / F_{2}$, Fahmi and Ripp (2010) pointed out an advantage and a drawback of the $\mathrm{AUC} / F_{2}$ approach. The advantage is that because $F_{2}$ can be visually evaluated just from the increase phase, its value can be estimated from an incomplete in vitro concentrationinduction curve. The drawback of the $F_{2}$ method is that when the potency of the induction response is very low, $F_{2}$ values are estimated in the region of saturated response, which is above $\mathrm{EC}_{50}$ and outside the definitively linear region of the concentration response. On the other hand, it is also possible to overcome the problems of limited dose-response curves from values in the initial slope. The use of the initial slope as an index of induction is clearly valid only if relevant in vivo drug concentrations are contained within the definitively 

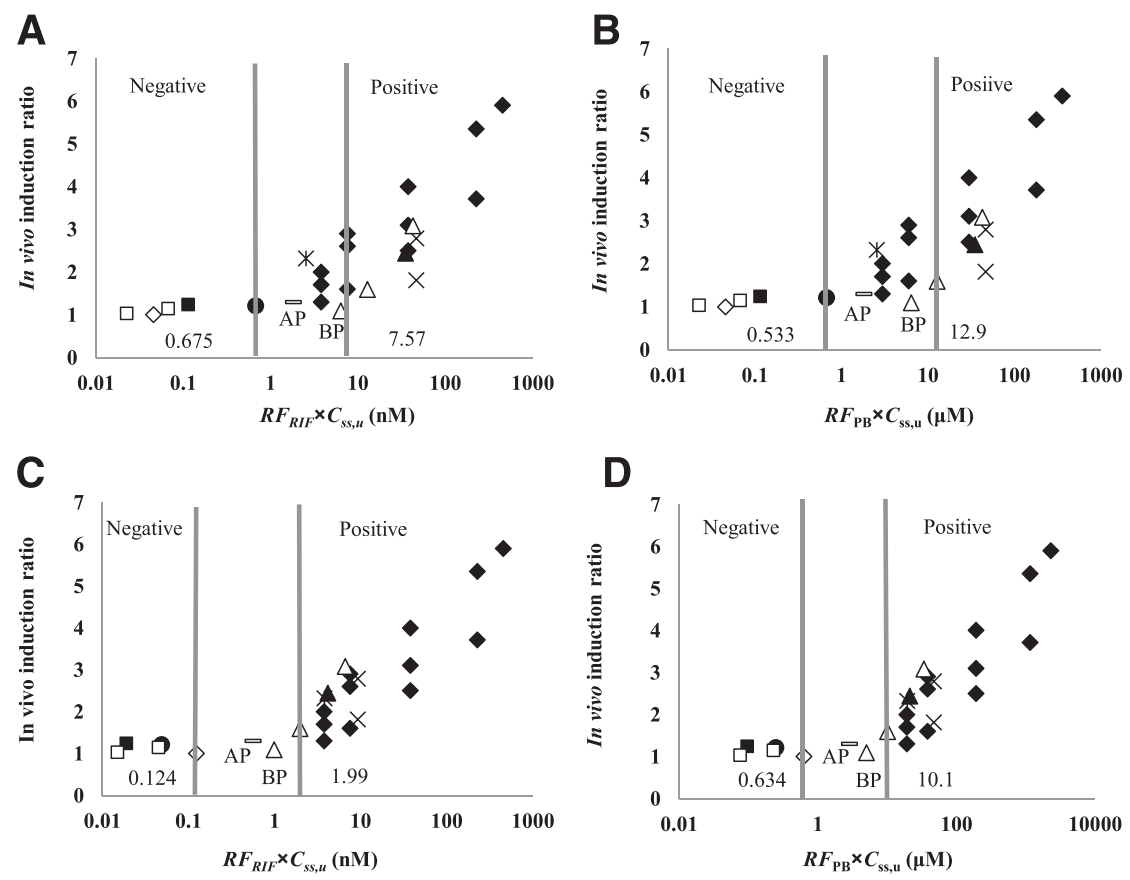

Fig. 4. (A-H) Relationship between $R F_{\mathrm{RIF}} \times C_{\mathrm{ss}, \mathrm{u}}$ (A, C, E, and $\mathrm{G})$ or $R F_{\mathrm{PB}} \times C_{\mathrm{ss}, \mathrm{u}}(\mathrm{B}, \mathrm{D}, \mathrm{F}$, and $\mathrm{H})$ and the in vivo CYP3A4 induction ratio (ratio of $\mathrm{CL}_{\text {int }}$ ) in humans for three lots of human hepatocytes [donor 1 (A and B), donor 2 (C and $\mathrm{D})$, donor 3 (E and F), and overall data (G and $\mathrm{H})]$. Gray lines denote the lower and upper thresholds by which negative and positive compounds are categorized. A lower threshold was assigned at the data point immediately preceding the point above 1.25 , and an upper threshold was assigned at the data point immediately after the point below 1.25. Closed diamond, RIF; closed triangle, CRB cross, PNT; asterisk, EFV; closed circle, PLC; horizontal bar, SLF; closed square, DXM; open diamond, NFD; open triangle, $\mathrm{PB}$; and open square, OMP. AP, point above 1.25; $\mathrm{BP}$, point below 1.25 .
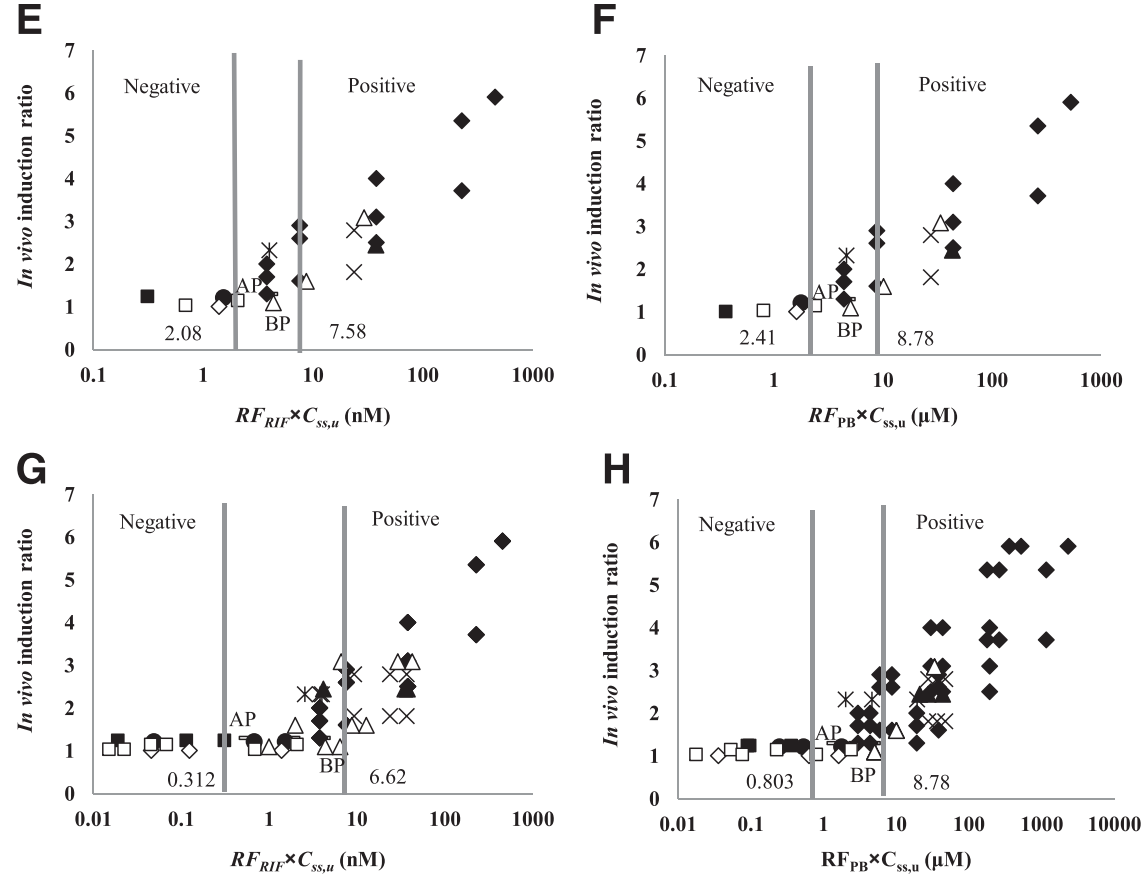

linear phase of the response curve (in other words, much lower than $\mathrm{EC}_{50}$ ) (Kato et al., 2005). The RF approach sufficiently meets this

governed by the $\mathrm{E}_{\max }$ model, and the reciprocal of the IDLC value corresponds to the initial slope of the induction response curve condition because the IDLC is minimal and definitely lower than $\mathrm{EC}_{50}$. In this region, the induction response shows linear increase as

(Fig. 1B). The RF value not only acts as the conversion factor to a standard inducer that elicits the same induction potential, but it also
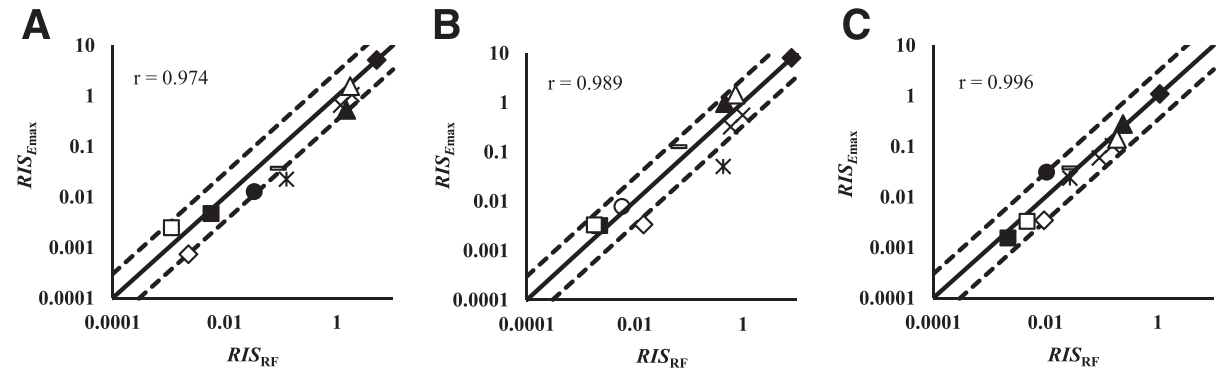

Fig. 5. (A-C) Correlation of RIS calculated using the calculated $E_{\max }$ and $\mathrm{EC}_{50}\left(\mathrm{RIS}_{E \max }\right)$ with RIS calculated using the $\mathrm{RF}_{\mathrm{RIF}}\left(\mathrm{RIS}_{\mathrm{RF}}\right)$ for three lots of human hepatocytes [donor $1(\mathrm{~A})$, donor 2 (B), and donor $3(\mathrm{C})$ ]. The solid line represents unity and dotted lines represent 3-fold line of unity. Closed diamond, RIF; closed triangle, CRB; cross, PNT; asterisk, EFV; closed circle, PLC; horizontal bar, SLF; closed square, DXM; open diamond, NFD; open triangle, $\mathrm{PB}$; and open square, OMP. 
acts as the ratio of the $\mathrm{EC}_{50}$ value of the test inducer to that of the standard inducer. Consequently, although the RF approach (like the $\mathrm{AUC} / F_{2}$ approach) uses only one parameter (IDLC), it can provide a robust and valid prediction of relevant in vivo induction potency.

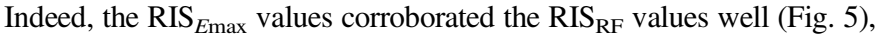
implying that the RF approach may be comparable with the $E_{\max }$ model approach.

In this analysis, the extent of intracellular exposure $\left(\mathrm{AUC}_{\text {intracell,u }}\right)$ was an important determining factor of the induction potency; therefore, we needed to be cautious when estimating the stability and unbound concentration in the culture medium. To confirm the stability in the culture medium, we evaluated the average concentrations of inducers and compared them with their theoretical concentrations (Supplemental Table 2). In this study, most of the inducers did not show a difference between the average and theoretical concentrations in the culture medium. However, the amounts of NFD, OMP, and PLC recovered in the induction experiments were low (Supplemental Table 2A). The low recovery of PLC might be attributed to plastic adsorption and metabolism (Sun et al., 2017). According to in-house data, NFD and OMP did not show the adsorption to plastic material (data not shown), so the low recovery of NFD and OMP might be attributed to metabolism. We should be cautious about interpreting the induction response for NFD, OMP, and PLC. The other important factor that accounted for $\mathrm{AUC}_{\text {intracell,u }}$ was the unbound fraction in the culture medium. The unbound fraction of most inducers was more than approximately 0.5 (Supplemental Table 3), and the theoretical concentrations of inducers corresponded to their intracellular unbound concentrations. Unfortunately, when we determined the induction of CYP3A4 by troglitazone (TRG) and analyzed TRG by the RF approach, it was only an outlier in the master curve (data not shown). TRG is well known for avidly binding to various proteins, which may reduce the unbound concentration in the culture medium substantially and cause the data points of TRG to deviate far from the curve. To accurately predict induction potential by the RF approach, it is essential to confirm the actual concentration of the test compound and its unbound fraction in the culture medium.

In summary, we demonstrate that evaluating data from cryopreserved human hepatocytes by the RF approach can robustly and relevantly predict the risk of DDI from CYP3A4 induction in a clinical setting. Furthermore, RF values obtained from IDLC values are very useful for adequately predicting the relevant in vivo induction potency when cellular toxicity and/or poor solubility of a new chemical entity gives a limited dose-induction response curve. Using the RF approach with cryopreserved hepatocytes can also provide a robust and relevant threshold for induction in the clinical setting that satisfies FDA and EMA guidelines. The simple RF approach using the IDLC value has been demonstrated to be a useful method to adequately assess the risk of CYP3A4 induction in humans.

\section{Acknowledgments}

The authors thank Sally Matsuura for helpful advice in the preparation and language editing of this paper.

\section{Authorship Contributions}

Participated in research design: Kuramoto, Kato, Shindoh, Kaneko. Conducted experiments: Kuramoto.

Performed data analysis: Kuramoto, Kato, Shindoh.

Wrote or contributed to the writing of the manuscript: Kuramoto, Kato, Shindoh, Kaneko, Ishigai, Miyauchi.

\section{References}

Chu V, Einolf HJ, Evers R, Kumar G, Moore D, Ripp S, Silva J, Sinha V, Sinz M, and Skerjanec A (2009) In vitro and in vivo induction of cytochrome p450: a survey of the current practices and recommendations: a Pharmaceutical Research and Manufacturers of America perspective. Drug Metab Dispos 37:1339-1354.

Fahmi OA and Ripp SL (2010) Evaluation of models for predicting drug-drug interactions due to induction. Expert Opin Drug Metab Toxicol 6:1399-1416.

Kanebratt KP and Andersson TB (2008) HepaRG cells as an in vitro model for evaluation of cytochrome P450 induction in humans. Drug Metab Dispos 36:137-145.

Kaneko A, Kato M, Endo C, Nakano K, Ishigai M, and Takeda K (2010) Prediction of clinical CYP3A4 induction using cryopreserved human hepatocytes. Xenobiotica 40:791-799.

Kaneko A, Kato M, Sekiguchi N, Mitsui T, Takeda K, and Aso Y (2009) In vitro model for the prediction of clinical CYP3A4 induction using HepaRG cells. Xenobiotica 39:803-810.

Kato M, Chiba K, Horikawa M, and Sugiyama Y (2005) The quantitative prediction of in vivo enzyme-induction caused by drug exposure from in vitro information on human hepatocytes. Drug Metab Pharmacokinet 20:236-243.

Ohtsuki S, Schaefer O, Kawakami H, Inoue T, Liehner S, Saito A, Ishiguro N, Kishimoto W, Ludwig-Schwellinger E, Ebner T, et al. (2012) Simultaneous absolute protein quantification of transporters, cytochromes P450, and UDP-glucuronosyltransferases as a novel approach for the characterization of individual human liver: comparison with mRNA levels and activities. Drug Metab Dispos 40:83-92.

Persson KP, Ekehed S, Otter C, Lutz ES, McPheat J, Masimirembwa CM, and Andersson TB (2006) Evaluation of human liver slices and reporter gene assays as systems for predicting the cytochrome p450 induction potential of drugs in vivo in humans. Pharm Res 23:56-69.

Ripp SL, Mills JB, Fahmi OA, Trevena KA, Liras JL, Maurer TS, and de Morais SM (2006) Use of immortalized human hepatocytes to predict the magnitude of clinical drug-drug interactions caused by CYP3A4 induction. Drug Metab Dispos 34:1742-1748.

Shimada T, Yamazaki H, Mimura M, Inui Y, and Guengerich FP (1994) Interindividual variations in human liver cytochrome P-450 enzymes involved in the oxidation of drugs, carcinogens and toxic chemicals: studies with liver microsomes of 30 Japanese and 30 Caucasians. J Pharmacol Exp Ther 270:414-423.

Sun Y, Chothe PP, Sager JE, Tsao H, Moore A, Laitinen L, and Hariparsad N (2017) Quantitative prediction of CYP3A4 induction: impact of measured, free, and intracellular perpetrator concentrations from human hepatocyte induction studies on drug-drug interaction predictions. Drug Metab Dispos 45:692-705.

Thelen K and Dressman JB (2009) Cytochrome P450-mediated metabolism in the human gut wall. $J$ Pharm Pharmacol 61:541-558.

Vermet H, Raoust N, Ngo R, Esserméant L, Klieber S, Fabre G, and Boulenc X (2016) Evaluation of normalization methods to predict CYP3A4 induction in six fully characterized cryopreserved human hepatocyte preparations and HepaRG cells. Drug Metab Dispos 44:50-60.

Zanger UM and Schwab M (2013) Cytochrome P450 enzymes in drug metabolism: regulation of gene expression, enzyme activities, and impact of genetic variation. Pharmacol Ther 138: $103-141$.

Zhang JG, Ho T, Callendrello AL, Clark RJ, Santone EA, Kinsman S, Xiao D, Fox LG, Einolf HJ, and Stresser DM (2014) Evaluation of calibration curve-based approaches to predict clinical inducers and noninducers of CYP3A4 with plated human hepatocytes. Drug Metab Dispos 42: 1379-1391.

Address correspondence to: Shino Kuramoto, Research Division, Chugai Pharmaceutical Co. Ltd., 200 Kajiwara, Kamakura, Kanagawa 247-8530, Japan. E-mail: kuramotosn@chugai-pharm.co.jp 